\title{
Procedures for screening out inaccurate reports of dietary energy intake
}

\author{
Megan A McCrory*, Cheryl L Hajduk and Susan B Roberts \\ The Energy Metabolism Laboratory, Jean Mayer USDA Human Nutrition Research Center on Aging at Tufts University, \\ 711 Washington Street, Boston, MA 02111 1-1524, USA
}

\begin{abstract}
Objective: To review existing methods and illustrate the use of a new, simple method for identifying inaccurate reports of dietary energy intake (rEI).

Design: Comparison of rEI with energy requirements estimated by using total energy expenditure predicted (pTEE) from age, weight, height and sex using a previously published equation. Propagation of error calculations was performed and cut-offs for excluding $\mathrm{rEI}$ at plus or minus two standard deviations ( $\pm 2 \mathrm{SD}$ ) and \pm 1 SD for the agreement between rEI and pTEE were established.

Setting: Dietary survey in a US national cohort: the Continuing Survey of Food Intakes by Individuals (CSFII), 1994-96.

Subjects: Men and non-pregnant, non-lactating women aged $21-45$ years in the CSFII who provided two multiple-pass 24-hour recalls, height and weight $(n=3755)$.

Results: Average rEI was 77\% of pTEE in men, and 64\% of pTEE in women. Calculated cut-offs were rEI $<40 \%$ or $>160 \%$ of pTEE ( \pm 2 SD) and $<70 \%$ or $>130 \%$ of pTEE ( $\pm 1 \mathrm{SD}$ ), respectively. Use of only the \pm 1 SD cut-offs, not the \pm 2 SD cut-offs, resulted in a relationship between rEI and body weight similar to what was expected (based on an independently calculated relationship between rEI and measured TEE). Exclusion of rEI outside either the \pm 2 SD (11\% of subjects) or \pm 1 SD ( $57 \%$ of subjects) cut-offs did not affect mean reported macronutrient intakes, but did markedly affect relationships between dietary composition and body mass index. Conclusions: When examining relationships between diet and health, use of $\pm 1 \mathrm{SD}$ cut-offs may be preferable to \pm 2 SD cut-offs for excluding inaccurate dietary reports.
\end{abstract}

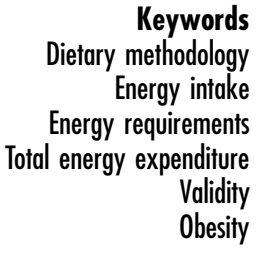

because of the high cost, it is not suitable for use in large dietary studies. Doubly labelled water theory and protocols for measuring TEE are discussed elsewhere ${ }^{15,16}$.

In this paper we review methods available for assessing inaccurate reports of dietary energy intake based on TEE assessed by doubly labelled water, as well as alternative techniques to use when doubly labelled water is not practical or available. In addition, we discuss the development of a new and simple method for screening out inaccurate reports of dietary energy intake that does not require doubly labelled water measurements of TEE and demonstrate its use with data from a recent US national dietary survey ${ }^{18}$. Finally, using this survey, we assess relationships between dietary components and body mass index (BMI) with and without the inclusion of inaccurate energy intake reports based on this new method. We find that different conclusions may be drawn depending on whether or not inaccurate reports are excluded from analyses of relationships between diet and health.

\section{Variance in dietary energy intake: implications for measuring actual vs. usual energy intake}

There is normally substantial day-to-day variation in food intake within an individual: average estimates for the 
within-subject coefficients of variation for daily energy intakes range between $23 \%$ and $31 \%{ }^{19,20}$. It has been estimated that dietary intake measurements are required for between 7 and 32 days, to classify correctly an individual's energy intake according to his or her true intake with $90 \%$ confidence ${ }^{21,22}$. Therefore, a dietary report obtained during any shorter-term dietary collection period could simply represent a small window within the normal variation of day-to-day food intake. In other words, it is possible that the report may represent the individual's actual intake during the recording period, but unless dietary reports are collected for at least 7 days, thereby capturing normal between-day variation, it may not represent habitual or usual intake (average energy intake required to maintain current body weight). For most studies, it is unrealistic to collect dietary information for more than 3-7 days (unless a food-frequency questionnaire (FFQ) is used), and large dietary surveys may have dietary information on even fewer days. However, given sufficient sample size, this may have little impact when the data are examined on a group basis because individual within-subject errors will probably be random and cancel out, thus providing a satisfactory estimate of the group mean ${ }^{22}$. In contrast, when dietary data are examined on an individual level, such as in regression analysis for examination of potential relationships between diet and health, caution should be exercised. The impact and implications of random within-subject error in this type of analysis were discussed recently ${ }^{23,24}$. These principles should be kept in mind when applying methods to detect and screen out individual underreports and overreports of dietary energy intake.

\section{Methods for identifying inaccurate energy intake reports}

\section{Separating under/over-recording and under/overeating}

Inaccurate energy intake reports can result from underrecording (defined as the failure to record all items and/or amounts consumed), undereating (defined as eating less than usual or than is required to maintain body weight), or a combination of both under-recording and undereating. Over-recording may also occur, though not commonly, and can result from failure to weigh leftover items not consumed during weighed dietary recording, or the tendency to overestimate items and amounts consumed during non-weighed recording and recall methods (i.e. 24hour recall and FFQ). It should also be noted that both overeating and undereating can also occur if subjects are not actually weight-stable but instead are in a phase of weight gain or loss, respectively. However, the methods discussed herein should only be used during periods of relative weight stability, i.e. when it is known that subjects are not actively gaining or losing weight prior to the measurement period.

Several investigators have used the simple difference between measured TEE and reported energy intake (or a ratio of the two) to identify under- and overreporters and quantify the extent of under- and overreporting. In order to determine the proportion of underreporting that is due to under-recording vs. undereating on a group basis, some investigators have also included measurement of body weight changes before and after the dietary collection period $^{10,25-29}$. The same combination of methods can also be used to determine this information for individuals; however, the application of this type of analysis for individuals may be limited by the fact that the combined within-subject measurement errors in dietary energy intake, body weight and energy requirements may exceed reporting errors in some individuals. In the case of underreporting, the difference between measured energy requirements and reported energy intake represents the degree of underreporting. If the group lost weight during the dietary measurement period, it can be assumed that energy equivalent of the weight loss is equal to the amount of energy by which the group underate. The difference between energy requirements and reported energy intake corrected for the change in body energy is equivalent to the amount of under-recording that occurred. Changes in body energy stores can be estimated from changes in body weight using an accurate and precise weight scale by assuming that $1 \mathrm{~kg}$ of body weight $=30 \mathrm{MJ}$ of energy ${ }^{11}$. Simple measurements of weight rather than complex measurements of body composition are used because methods currently available for measuring changes in body composition are not precise enough to measure small changes in fat mass and fat-free mass. Therefore, unless the weight loss (and therefore undereating) is sufficiently large, changes in body energy stores cannot be quantified accurately from measured changes in body composition.

Goris et al. ${ }^{10}$ and Bathalon et $a l^{28}$ used similar techniques to determine the extent of underreporting that could be attributed to undereating vs. underrecording. Both groups of investigators used doubly labelled water to determine energy requirements, and measured body weight before and after the dietary recording period. The change in body energy was calculated using the factor of $0.03 \mathrm{MJ}(7 \mathrm{kcal})$ per gram of weight change. Goris et $a l^{10}$ found that middle-aged obese men (mean $\mathrm{BMI}=34 \pm 4 \mathrm{~kg} \mathrm{~m}^{-2}$ ) reported an average energy intake of only $62 \%$ of TEE using 7 -day, non-weighed intake records; therefore, underreporting occurred by an average of approximately $38 \%$. In addition, body weight loss averaged $1.0 \mathrm{~kg}$, leading the investigators to conclude that the group underate by $26 \%$ and underrecorded by $12 \%$, the latter of which was also verified by changes in water balance.

In the study by Bathalon et al. ${ }^{28}$, under-recording and 
undereating were assessed in restrained and unrestrained postmenopausal women (as measured by the Eating Inventory ${ }^{30}$ ) group-matched for body composition. Restrained eaters reportedly practise weight control by consciously restricting their intake of certain food items, and also typically have a lower rEI for a given body weight compared with unrestrained eaters ${ }^{28}$. However, it was previously unknown if the relatively low $\mathrm{rEI}$ in restrained subjects was due to actual lower energy requirements or to dietary underreporting. Using the average intake calculated by three dietary methods (7-day weighed report, 24hour recall and FFQ), the researchers found that both groups of women underreported energy intake: the unrestrained eaters by $17 \%$ and the restrained eaters by $23 \%$. From changes in body energy it could also be determined that, in the unrestrained eaters, the majority of underreporting was due to undereating (16\%), while in the restrained eaters, about half (12\%) of the underreporting was due to undereating and half (11\%) was due to the failure to record certain food items or amounts eaten. Therefore, these restrained eaters did not in fact have lower energy requirements than their unrestrained counterparts; furthermore, restrained eaters is one group of subjects that as a whole might not be expected to record all food items consumed during dietary assessment.

It is difficult to determine whether the differences in the magnitude of under-recording (38\% vs. 17-23\%) between the above two studies was primarily due to the different dietary intake methods used, the different sexes, eating behaviours, degrees of obesity, or other factors. Goris et $a l .{ }^{10}$ used a 7-day estimated rather than a weighed record, and of the three dietary methods used by Bathalon et $a l^{28}$, the FFQ resulted in the greatest degree of underrecording in both restrained and unrestrained groups while the 7-day weighed report resulted in the least degree of under-recording. Bathalon et $a l^{28}$ also reported that subjects who experienced less hunger as measured by the Eating Inventory ${ }^{30}$, independent of restraint and disinhibition scores, were more likely to underreport energy intake. In other studies that did not distinguish between under-recording and undereating, reporting errors have been associated with a number of different subject characteristics including sex, BMI, age, ethnicity, race and cultural factors, physical activity, smoking status, education level, literacy, social class, living arrangements, depression, past dieting frequency and dietary restraint $t^{5,8,31-36}$. The association between many of these factors and underreporting is reviewed by Macdiarmid and Blundell ${ }^{37}$.

\section{Goldberg cut-offs}

One of the most widely used procedures for identifying inaccurate reports of energy intake is the method first developed by Goldberg and co-workers ${ }^{38}$. This method assesses the validity of rEI by comparing TEE with rEI when both are expressed as a multiple of basal metabolic rate (BMR). In other words, during weight stability,
$\mathrm{rEI} / \mathrm{BMR}=\mathrm{TEE} / \mathrm{BMR}$. The TEE/BMR ratio is also known as the physical activity level (PAL); so the equation can be rewritten as $\mathrm{rEI} / \mathrm{BMR}=\mathrm{PAL}$. Physiologically, average PAL varies among groups from 1.2 for those chair-bound or bedridden, to 1.6-1.9 for those doing moderate work, to a maximum of about $2.4-2.8$ for soldiers on active duty and professional and amateur athletes ${ }^{39}$.

Two cut-offs for the agreement between PAL and rEI/BMR were developed by Goldberg et $a l^{38}$ and their application was first demonstrated by Black et al. ${ }^{40}$. 'CUTOFF 1' was set at a PAL of 1.35 , representing a minimum plausible value for weight maintenance for most individuals (except those chair-bound or bedridden). Therefore, using CUT-OFF 1, values of rEI/BMR less than 1.35 would be considered as having poor validity because it is unlikely that most individuals would be able to maintain weight with a usual energy intake below this minimum level. However, Black ${ }^{20}$ recently recommended that CUT-OFF 1 no longer be used to identify inaccurate reports of energy intake because it ignores biological variability and measurement errors for both energy intake and TEE. In addition, its use leads to underestimation of the prevalence of underreporting when used for individuals whose daily activity is above a sedentary level. Therefore, for the remainder of this paper, in mentioning the Goldberg cut-off, we will be referring specifically to the second cut-off, 'CUT-OFF 2', which is explained in the following paragraph.

CUT-OFF 2 differs from CUT-OFF 1 in that its value varies depending on the actual or expected TEE (or PAL) of the population or individuals under study. It also involves a statistical comparison between rEI/BMR and PAL, taking account of both biological variability and measurements errors. In the original paper showing the derivation of CUT-OFF 2, only a lower cut-off was developed because it was calculated with the assumption that subjects were sedentary. But as explained recently by Black $^{20}$, when the activity level is known or can be presumed based on available information, an upper limit can also be calculated. Lower and upper limits for CUTOFF 2 are derived via a statistical comparison between $\mathrm{rEI} / \mathrm{BMR}$ and PAL and represent the 95\% lower and upper confidence limits, respectively, for the difference between rEI/BMR and PAL. Therefore, the actual values for the upper and lower limits of CUT-OFF 2 can differ depending on the activity level of the individuals being studied. As explained by Black ${ }^{20}$, the upper and lower limits of CUTOFF 2 for detecting under- and overreporting are given by:

$$
\mathrm{rEI} / \mathrm{BMR}>\mathrm{PAL} \times \exp \left[\mathrm{SD}_{\min } \times((S / 100) / \sqrt{n})\right]
$$

(lower limit)

and

$$
\mathrm{rEI} / \mathrm{BMR}<\mathrm{PAL} \times \exp \left[\mathrm{SD}_{\max } \times((S / 100) / \sqrt{n})\right]
$$

(upper limit) 
where SD is standard deviation and, for a 95\% confidence interval for the comparison between $\mathrm{rEI} / \mathrm{BMR}$ and $\mathrm{PAL}, \mathrm{SD}_{\min }=-2$ and $\mathrm{SD}_{\max }=+2$. The number of subjects in the study is denoted by $n$; but $n=1$ when these formulae are used to detect under- and overreporting in individuals. $S$ is the factor that accounts for variation in EI, BMR and energy requirements, and is calculated by:

$$
S=\sqrt{\left(\mathrm{CV}_{\mathrm{wEI}}^{2} / d\right)+\mathrm{CV}_{\mathrm{wB}}^{2}+\mathrm{CV}_{\mathrm{tP}}^{2}}
$$

where $\mathrm{CV}_{\mathrm{wEI}}$ is the within-subject coefficient of variation in energy intake, $d$ is the number of days of energy intake measurement, $\mathrm{CV}_{\mathrm{wB}}$ is the precision of BMR measurement or estimation and $\mathrm{CV}_{\mathrm{tP}}$ is the total variation in PAL. Full derivation and a thorough explanation of the above formulae are given by Goldberg et l $^{38}$.

While it is important to identify inaccurate reports of energy intake, as noted by $\mathrm{Black}^{20}$, the use of the Goldberg cut-off for doing so has marked limitations. Most notably, in order to use the Goldberg cut-off, it is necessary to make an assumption of a certain PAL for each individual. That is, habitual activity level, or energy requirements, must be known in order to assign an appropriate PAL. However, the error in assigning PAL is one source of variability that is not accounted for by Goldberg et $a l^{38}$ or Black ${ }^{20}$ in their analyses. Based on published data ${ }^{41}$, we estimated that even when PAL is calculated by factorial analysis from detailed time-motion records, the within-subject coefficient of variation for the agreement between this calculated PAL and TEE/BMR, where TEE is measured by doubly labelled water, is $15 \%$. This low precision and therefore accuracy in the assignment of a PAL for individual subjects is likely to be one reason for the lack of sensitivity of the Goldberg cut-off for identifying inaccurate energy intake reports. Black recently performed sensitivity and specificity analysis and showed that when individual PALs are assigned according to World Health Organization categories ${ }^{42}$, while the specificity of the Goldberg cut-off for including all accurate reports of energy intake was high (0.97 for men and 0.98 for women), its sensitivity was relatively low, at 0.76 and 0.85 for men and women, respectively ${ }^{17}$. A third limitation of the Goldberg cut-off is that although both underreporting and overreporting can occur to varying degrees, it only identifies extremely inaccurate reporting (i.e. $<2$ SD for the agreement between $\mathrm{rEI} / \mathrm{BMR}$ and PAL). This may be one reason why some investigators have used the percentage difference between $\mathrm{rEI}$ and either measured or predicted TEE to identify inaccurate reports and determine the degree of misreporting in individuals. However, this method is technically incorrect when applied to individual reports because it does not take into account any errors in the methods used to quantify TEE and rEI. $\pm 1 S D$ and \pm 2 SD cut-offs based on predicted TEE In an attempt to overcome some of the problems associated with using the Goldberg cut-off discussed above, we developed an alternative approach for identifying inaccurate records of dietary energy intake, in part based on the reasoning outlined by Goldberg et $a l^{38}$ and more recently by Black $^{20}$. While our method uses the percentage difference between TEE predicted from published equations (pTEE) and rEI, it also takes into account the within-subject errors in these parameters. Since TEE is predicted, use of this method should theoretically eliminate the potential error of assigning inaccurate PALs with only limited information on the activity of individuals under study. Furthermore, since pTEE is based on the simple parameters of age, weight, height and sex, it can be used when there is little or no information available to help investigators assign an appropriate PAL.

The TEE prediction equation of Vinken et $a l^{43}$ was recently developed using data from 93 subjects who participated in free-living doubly labelled water studies, and cross-validated in two external samples. The subjects ranged in age from 18 to 81 years and BMI ranged from 18 to $32 \mathrm{~kg} \mathrm{~m}^{-2}$. Measured TEE varied from 12.3 to 18.6 $\mathrm{MJ} \mathrm{day}^{-1}$, and PAL derived from measured TEE and BMR ranged from 1.2 to 2.6. The equation is as follows:

$$
\begin{aligned}
\text { pTEE }= & 7.377-0.073 \times \text { age }+0.0806 \times \text { weight } \\
& +0.0135 \times \text { height }-1.363 \times \text { sex }
\end{aligned}
$$

where age is in years, weight is in kg, height is standing height in $\mathrm{cm}$, and sex is 0 for men and 1 for women. The equation has an $R^{2}$ of 0.64 and standard error of the estimate (SEE) of $1.8 \mathrm{MJ} \mathrm{day}^{-1}$, but bootstrap analysis showed that the true SEE of the equation is $1.9 \mathrm{MJ} \mathrm{day}^{-1}$. This represents $17.7 \%$ of the mean measured TEE by doubly labelled water of the 93 subjects.

To illustrate the use of pTEE for identifying inaccurate reports of dietary energy intake, we applied our procedures to a recent US national survey, the Continuing Survey of Food Intakes by Individuals, 1994-96 (CSFII $1994-96)^{18}$. Briefly, the CSFII is a representative sample of 16303 non-institutionalised persons, defined by age, sex and income level. Participants provided one or two 24hour recalls of dietary intake and self-reported weight and height. For this analysis, we used data from CSFII men and non-pregnant, non-lactating women aged 21-45 years, who provided 2 days of dietary intake, as well as selfreported weight and height $(n=3755)$.

We calculated \pm 1 SD and \pm 2 SD cut-offs for the agreement between rEI and pTEE based on principles outlined by $\mathrm{Black}^{20}$, where

$$
\begin{aligned}
\pm 1 \mathrm{SD} & =\sqrt{\left(\mathrm{CV}_{\mathrm{wEI}}^{2} / d\right)+\mathrm{CV}_{\mathrm{wTEE}}^{2}} \\
& =\sqrt{\left(\mathrm{CV}_{\mathrm{wEI}}^{2} / d\right)+\mathrm{CV}_{\mathrm{wpTEE}}^{2}+\mathrm{CV}_{\mathrm{tmTEE}}^{2}},
\end{aligned}
$$


using values of $8.2 \%$ for $\mathrm{CV}_{\text {tmTEE}}$, which includes the technical error of measuring TEE by the doubly labelled water method as well as biological variation ${ }^{20}$, and $17.7 \%$ for $\mathrm{CV}_{\text {wpTEE }}{ }^{43}$. Errors for both measured and predicted TEE are included in the above equation because the errors in prediction of TEE are in part dependent upon the error of measuring TEE. As Black discusses, either a standard $\mathrm{CV}_{\text {wEI }}$ of $23 \%$ can be used, or a value specific to the dataset being analysed can be substituted as appropriate. We calculated $\mathrm{CV}_{\mathrm{wEI}}$ for the above-defined 3755 individuals in CSFII to be $31.2 \%$, and used 2 for $d$ because there are two 24-hour recalls. Using the above formula, the \pm 1 SD for the agreement between $\mathrm{rEI}$ and pTEE is $\pm 29.4 \%$; therefore, we used the close approximation of $\pm 30 \%$ for the \pm 1 SD cut-offs and $60 \%$ for the \pm 2 SD cut-offs. In other words, using the \pm 1 SD cut-offs means that individuals with an rEI of $<70 \%$ or $>130 \%$ difference from pTEE would be identified as underreporters and overreporters, respectively. Similarly, individuals with an rEI of $<40 \%$ or $>160 \%$ difference from pTEE would be identified as inaccurate reporters as defined by the \pm 2 SD cut-offs.

It should be noted that Black $^{20}$ and Goldberg et al. ${ }^{38}$ only calculate and use \pm 2 SD cut-offs (i.e. the $95 \%$ confidence limits) and do not use \pm 1 SD cut-offs, arguing that the identification of inaccurate reporters should be based on a standard statistical comparison because while the measured energy intake may not represent habitual intake, it could still represent actual intake during the measurement period. However, it is possible that, in certain situations, it could be important to screen out those energy intake reports that are not representative of usual energy intake even though the reports may be representative of what was actually consumed during the measurement period (e.g. when studying relationships between habitual dietary intake and health outcomes).

The following analysis illustrates use of the $\pm 1 \mathrm{SD}$ and \pm 2 SD cut-off levels for screening out inaccurate energy intake reports, and compares the impact of applying these different cut-offs vs. using no cut-offs on several outcomes of interest using the above-defined subset of the CSFII dataset $(n=3755)$. Mean \pm SD values for $\mathrm{rEI}$ and $\mathrm{pTEE}$ are shown in Table 1. pTEE for the men and women (14.1 and $11.3 \mathrm{MJ} \mathrm{day}^{-1}$, respectively) are similar to expected values for this age range ${ }^{44}$. The rEI for both men and women was significantly lower than pTEE, with rEI being only $77 \%$ of pTEE in men and 64\% of pTEE in women. The Recommended Dietary Allowance (RDA) for energy is also shown for comparison because rEI as a percentage of RDA is a variable provided in the CSFII dataset. The rEI was also significantly lower than the RDA, but was only $89 \%$ and $77 \%$ of the RDA for men and women, respectively. The discrepancy between the degree of underreporting when rEI is compared with pTEE vs. RDA is due to the fact that the energy RDAs for adults are too low because they are based
Table 1 Reported energy intake ( $\mathrm{rEl}$ ), predicted total energy expenditure (pTEE) and the US Recommended Dietary Allowance (RDA) for energy in men and non-pregnant, non-lactating women aged 21-45 years from the Continuing Survey of Food Intakes by Individuals 1994-96 $(n=3755)$. Results are presented as mean \pm standard deviation

\begin{tabular}{|c|c|c|}
\hline & $\operatorname{Men}(n=1969)$ & Women $(n=1786)$ \\
\hline rEl (MJ day $\left.{ }^{-1}\right)$ & $10.8 \pm 4.1^{*}$ & $7.1 \pm 2.5^{\star}$ \\
\hline $\mathrm{rEl} / \mathrm{pBMR}$ & $1.38 \pm 0.53$ & $1.12 \pm 0.44$ \\
\hline \multicolumn{3}{|l|}{ PTEE comparison } \\
\hline pTEE (MJ day ${ }^{-1}$ ) & $14.1 \pm 1.4$ & $11.3 \pm 1.5$ \\
\hline rEl/pTEE (\%) & $77 \pm 29$ & $64 \pm 24$ \\
\hline PAL $_{\text {pTEE }}$ (pTEE/pBMR) & $1.79 \pm 0.06$ & $1.87 \pm 0.08$ \\
\hline \multicolumn{3}{|l|}{ RDA comparison } \\
\hline RDA (MJ day ${ }^{-1}$ ) & $12.1 \pm 0.0^{\star \star}$ & $9.2 \pm 0.0^{* \star}$ \\
\hline $\mathrm{rEI} / \mathrm{RDA}\left(\mathrm{MJ}\right.$ day $^{-1}$ ) & $89 \pm 34$ & $77 \pm 28$ \\
\hline $\mathrm{PAL}_{\mathrm{RDA}}(\mathrm{RDA} / \mathrm{pBM} \mathrm{R})$ & $1.56 \pm 0.16$ & $1.55 \pm 0.17$ \\
\hline
\end{tabular}

pBMR - predicted basal metabolic rate; PAL - physical activity level. * Significantly different from $\mathrm{PTEE}$ and RDA, $P<0.01$.

** Significantly different from pTEE, $P<0.001$.

on methods for measuring energy expenditure that may underestimate $\mathrm{TEE}^{45,46}$.

To identify individual underreports and overreports, we compared each subject's rEI/pTEE value to the \pm 2 SD cutoffs of rEI $<40 \%$ and $>160 \%$ of pTEE, and the \pm 1 SD cut-offs of $<70 \%$ and $>130 \%$ of pTEE. Using the \pm 2 SD cut-offs, $89 \%$ of the subjects reported accurately, while using the \pm 1 SD cut-offs, only $43 \%$ of the subjects reported accurately. This is illustrated in Fig. 1. To determine whether the degree of misreporting was associated with BMI, we plotted the relationship between $\mathrm{rEI} / \mathrm{pTEE}(\%)$ and BMI, also shown in Fig. 1. In general, underreporting occurred throughout the entire BMI range, while overreporting occurred only at BMI $<40 \mathrm{~kg} \mathrm{~m}^{-2}$. However, consistent with other studies, the majority of misreporting was due to underreporting rather than overreporting.

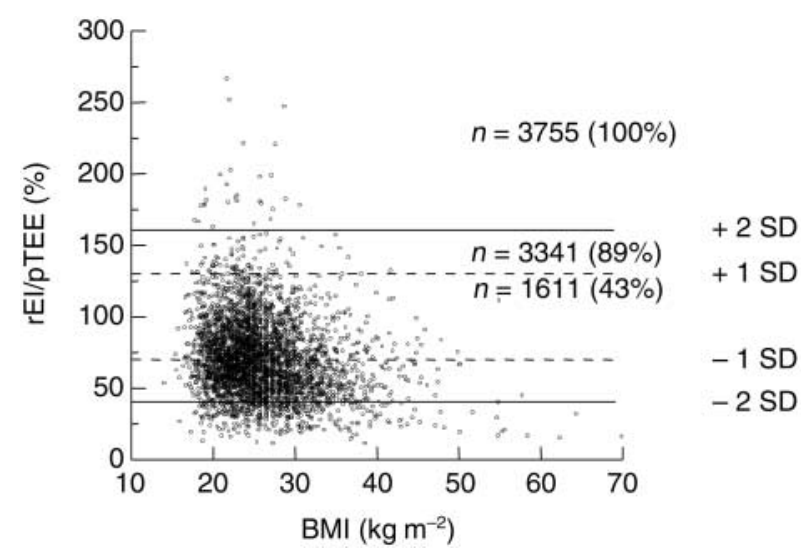

Fig. 1 The degree of misreporting (indicated as reported energy intake/predicted total energy expenditure (rEl/pTEE (\%))) as a function of body mass index (BMI) in $n=3755$ men and nonpregnant, non-lactating women aged 21-45 years who participated in the Continuing Survey of Food Intakes by Individuals 1994-96. The outer and inner solid and dotted lines represent the $\pm 2 \mathrm{SD}$ and $\pm 1 \mathrm{SD}$ cut-offs, respectively, for the agreement between rEl and pTEE 
On the basis of energy balance principles and the reported relationship between TEE measured by doubly labelled water and body weight ${ }^{47,48}$, habitual energy intake should increase with increasing body weight. We therefore plotted the relationship between rEI and body weight for the entire sample $(n=3755)$, the accurate reporters as defined by the \pm 2 SD cut-offs $(n=3341)$ and the accurate reporters as defined by the \pm 1 SD cut-offs ( $n=1611)$, as shown in Fig. 2 . We also plotted the same relationship in $n=1611$ subjects randomly selected (by computer) for comparison. In addition, we calculated the expected relationship between rEI and body weight by determining the relationship between measured TEE (mTEE, by doubly labelled water) and body weight using the 93 subjects from the study by Vinken et $a l^{43}$. Thus, if the cut-offs are working as predicted to screen out the inaccurate reporters while leaving in primarily the accurate reporters, then the slope of the relationship between rEI and body weight should be similar to that of the relationship between mTEE and body weight. For each panel shown in Fig. 2, the solid line represents the regression line for the relationship between body weight and $\mathrm{rEI}$, while the dotted line represents the regression line for the relationship between body weight and mTEE $\left(\right.$ mTEE, MJ day ${ }^{-1}=3.509+0.107 \times$ body weight, $\mathrm{kg}$; adj $\left.R^{2}=0.20, \mathrm{SEE}=2.61 \mathrm{MJ} \mathrm{day}^{-1}, P<0.001\right)$. While the relationship between body weight and rEI was significant in all cases $(P<0.00001)$, as expected due to the high degree of underreporting, the slope of the relationship between rEI and body weight was the lowest when no exclusions were made $($ slope $=0.047)$. The slope increased to 0.067 when misreporters outside the $\pm 2 \mathrm{SD}$ cut-offs were excluded, and increased even more to 0.097 when misreporters outside the \pm 1 SD cut-offs were excluded. As can be seen in Fig. 2, this slope of 0.097 of the relationship between body weight and rEI was very close to the slope of the relationship between body weight and mTEE (0.107). Finally, the slope of the relationship between body weight and rEI was only 0.051 when using the randomly selected subjects $(n=1611)$, a value very close to the slope of 0.047 for this relationship using the entire sample $(n=3755)$. The combination of these comparisons suggests that a \pm 1 SD cut-off may more effectively exclude individuals failing to report usual dietary intake than either a \pm 2 SD cut-off or no exclusions, and may therefore be best suited for use in studies requiring analysis of individual dietary data.

We were also interested in examining the impact of using the different cut-offs on reported dietary composition. Table 2 shows mean \pm standard error of the mean for BMI, rEI, macronutrients and fibre in the entire sample, in the accurate reporters defined by the $\pm 2 \mathrm{SD}$ and $\pm 1 \mathrm{SD}$ cut-offs, and in the randomly selected subjects. Mean rEI increased somewhat, from $71 \%$ of pTEE in the entire sample to $74 \%$ of pTEE when using the \pm 2 SD cut-offs, and substantially to $90 \%$ of pTEE when using the \pm 1 SD cut-offs. In the random sample, mean rEI was identical to that in the entire sample. There were no major alterations
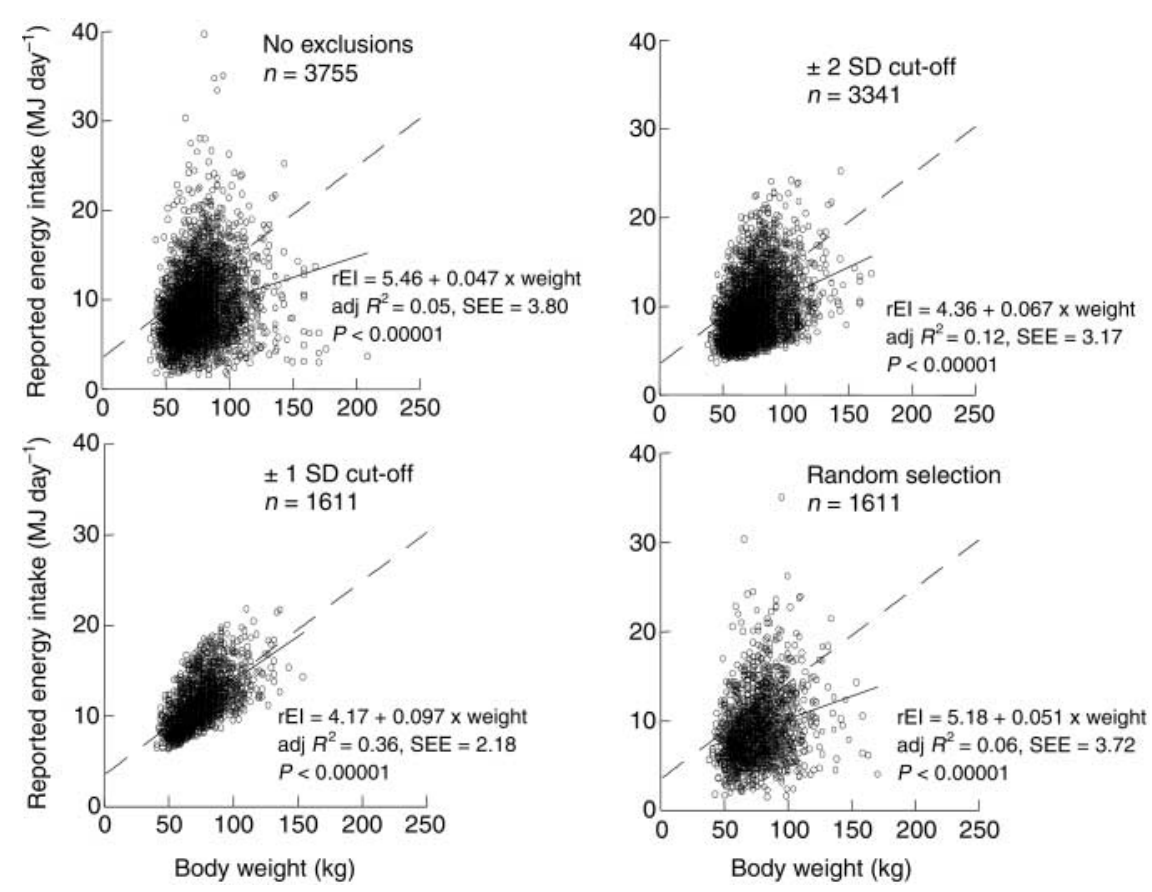

Fig. 2 Relationship between reported energy intake ( $\mathrm{rEl}$ ) and body weight in the full subset who participated in the Continuing Survey of Food Intakes by Individuals 1994-96, using the two cut-off levels, and in a random sample. Solid lines represent the regression lines for the relationship between $\mathrm{rEI}$ and body weight. Dotted lines represent the relationship between rEI and total energy expenditure measured (mTEE) by doubly labelled water using data from Vinken et al. ${ }^{43}$, and indicate the expected relationship between rEI and body weight $\left(\mathrm{mTEE}, \mathrm{MJ}\right.$ day $^{-1}=3.509+0.107 \times$ body weight, $\mathrm{kg} ;$ adj $\left.R^{2}=0.20, \mathrm{SEE}=2.61 \mathrm{MJ}^{2} \mathrm{day}^{-1}, P<0.001\right)$ 
Table 2 Dietary intake and body mass index (BMI) by energy intake reporting accuracy group in adults aged 21-45 years from the Continuing Survey of Food Intakes by Individuals 1994-96. The results are presented as mean \pm standard error of the mean

\begin{tabular}{|c|c|c|c|c|}
\hline & $\begin{array}{c}\text { All } \\
(n=3755)\end{array}$ & $\begin{array}{c} \pm 2 \mathrm{SD} \\
(n=3341)\end{array}$ & $\begin{array}{c} \pm 1 \mathrm{SD} \\
(n=1611)\end{array}$ & $\begin{array}{c}\text { Random } \\
(n=1611)\end{array}$ \\
\hline BMI $\left(\mathrm{kg} \mathrm{m}^{-2}\right)$ & $26.0 \pm 0.1$ & $25.7 \pm 0.1$ & $25.0 \pm 0.1$ & $25.9 \pm 0.1$ \\
\hline rEl (MJ day $\left.{ }^{-1}\right)$ & $9.0 \pm 0.1$ & $9.5 \pm 0.1$ & $11.5 \pm 0.1$ & $9.0 \pm 0.1$ \\
\hline rEI (\% pTEE) & $71 \pm 0$ & $74 \pm 0$ & $90 \pm 0$ & $71 \pm 1$ \\
\hline Fat (\% energy) & $33.4 \pm 0.1$ & $33.4 \pm 0.1$ & $34.2 \pm 0.2$ & $33.5 \pm 0.2$ \\
\hline Carbohydrate (\% energy) & $50.0 \pm 0.2$ & $49.9 \pm 0.2$ & $49.2 \pm 0.2$ & $49.9 \pm 0.2$ \\
\hline Protein (\% energy) & $15.6 \pm 0.1$ & $15.4 \pm 0.1$ & $14.8 \pm 0.1$ & $15.7 \pm 0.1$ \\
\hline Alcohol (\% energy) & $2.2 \pm 0.1$ & $2.3 \pm 0.1$ & $2.9 \pm 0.2$ & $2.1 \pm 0.2$ \\
\hline Fibre $\left(\mathrm{gMJ}^{-1}\right)$ & $1.8 \pm 0.0$ & $1.8 \pm 0.0$ & $1.7 \pm 0.0$ & $1.8 \pm 0.0$ \\
\hline
\end{tabular}

rEI - reported energy intake; pTEE - predicted total energy expenditure.

in mean reported dietary composition when using either of the two cut-offs vs. the entire sample.

We also examined dietary composition predictors of BMI using the entire sample, the two cut-offs, and the random sample (Table 3). In each case, the potential predictors examined were the percentage of energy from dietary fat and total fibre normalised for energy intake, because controversy still exists over the relative contribution of each to weight control and the development of obesity. We performed stepwise multiple regression analysis, and in each case controlled for age, sex, current smoking status and hours per day of television viewing. As shown in the table, when either the entire sample or the random sample was used, only the percentage of energy from dietary fat was a significant predictor of BMI. When reports outside the \pm 2 SD cut-offs were excluded, the percentage of energy from dietary fat and fibre independently predicted BMI in the same model. When the \pm 1 SD cut-offs were used, again both dietary fat and fibre entered the model, but the coefficients for both were higher than the respective coefficients when the $\pm 2 \mathrm{SD}$ cut-offs were used and the adjusted $R^{2}$ increased while the SEE decreased. This analysis demonstrates that inaccurate reports of dietary energy intake can obscure relationships between diet and health, and in the case of energy regulation, may lead to underestimation of the importance of low dietary fibre and high dietary fat in the maintenance of excess weight. These findings are in general agreement with those of Macdiarmid et al. ${ }^{49}$ and Stallone et al. ${ }^{50}$, who also found different relationships between nutritional parameters and BMI, and dietary intake and socioeconomic status, respectively, with and without the exclusion of 'low energy reporters' as defined by using the Goldberg cut-off (using a CUT-OFF 1 value of $\mathrm{EI} / \mathrm{BMR}<1.2$ ).

\section{Validation of self-assessed usual intake}

We also tested whether self-reported usual intake was valid when compared to either the \pm 1 SD or \pm 2 SD cutoffs for the agreement between rEI and pTEE. As part of the CSFII 24-hour recall procedure, regarding their energy intake, participants were asked to respond whether they ate their usual amount, ate more than usual or less than usual. As shown in Fig. 3, according to the \pm 2 SD cut-offs, $90 \%$ of those who said they consumed their 'usual amount' reported accurately (i.e. actually did eat their usual amount). However, according to the more stringent \pm 1 SD cut-offs, only $44 \%$ reported accurately while $54 \%$ underreported (i.e. actually ate less than usual). This suggests that individuals' self-defined 'usual amount' ingested may actually be within the range of normal wide variations range of in day-to-day intake, but this may not be the same

Table 3 Evaluation of best-fit models for macronutrient predictors of body mass index by energy intake reporting accuracy group in adults aged 21-45 years from the Continuing Survey of Food Intakes by Individuals 1994-96. In each reporting accuracy group, dietary fat percentage and fibre were examined as potential predictors, and all models are controlled for age, sex, current smoking status and hours per day of television viewing

\begin{tabular}{|c|c|c|c|c|c|}
\hline & \multicolumn{3}{|c|}{ Predictor statistics } & \multicolumn{2}{|c|}{ Model statistics } \\
\hline & $\beta$ coefficient (SE) & Partial $r$ & $P$-value & $\operatorname{adj} R^{2}$ & SEE \\
\hline \multicolumn{6}{|l|}{ All $(n=3755)$} \\
\hline $\begin{array}{c}\text { Fat (\% energy) } \\
\pm 2 \text { SD }(n=3341)\end{array}$ & $0.043(0.010)$ & 0.063 & 0.0001 & 0.053 & 5.30 \\
\hline $\begin{array}{c}\text { Fat (\% energy) } \\
\text { Fibre }(\mathrm{g} \mathrm{MJ}-1) \\
\pm 1 \mathrm{SD}(n=1611)\end{array}$ & $\begin{array}{r}0.051(0.011) \\
-112.45(28.05)\end{array}$ & $\begin{array}{r}0.077 \\
-0.069\end{array}$ & $\begin{array}{l}<0.0001 \\
<0.0001\end{array}$ & 0.069 & 4.83 \\
\hline $\begin{array}{l}\text { Fat }(\% \text { energy) } \\
\text { Fibre }\left(\mathrm{g} \mathrm{MJ}^{-}{ }^{1}\right)\end{array}$ & $\begin{array}{r}0.073(0.015) \\
-141.87(39.86)\end{array}$ & $\begin{array}{r}0.118 \\
-0.089\end{array}$ & $\begin{array}{r}<0.0001 \\
0.0004\end{array}$ & 0.093 & 4.41 \\
\hline Random $(n=1611)$ & & & & & \\
\hline Fat (\% energy) & $0.047(0.016)$ & 0.071 & 0.005 & 0.059 & 5.01 \\
\hline
\end{tabular}

SE - standard error; SEE - standard error of the estimate. 
880

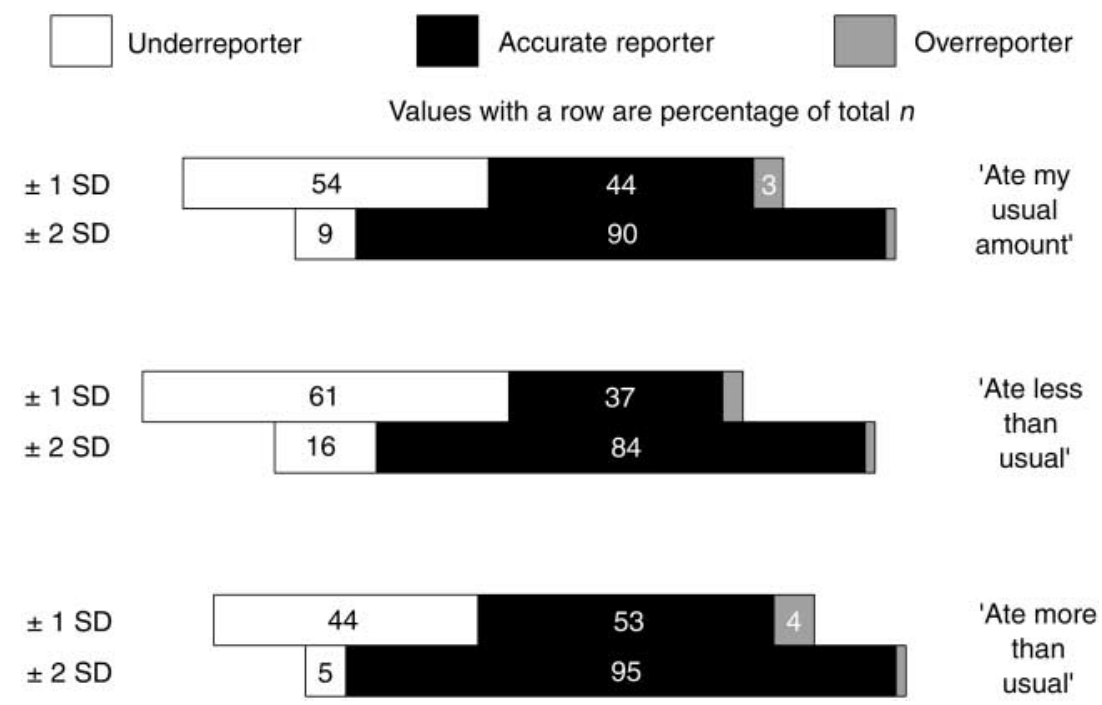

Fig. 3 Agreement between subjective and objective measures of usual energy intake in men and non-pregnant, non-lactating women aged 21-45 years who participated in the Continuing Survey of Food Intakes by Individuals 1994-96. Self-assessed usual intake was compared with usual intake assessed by $\pm 2 \mathrm{SD}(n=3341)$ and \pm 1 SD $(n=1611)$ cut-offs

as the 'habitual' amount needed to maintain current body weight.

Of the individuals who reported consuming 'less than usual', according to the \pm 2 SD cut-offs, only $16 \%$ underreported (i.e. actually ate less than usual) and $84 \%$ reported accurately (i.e. actually consumed a usual amount), whereas according to the \pm 1 SD cut-offs, $61 \%$ indeed underreported accurately (i.e. actually did consume less than usual) and only $37 \%$ reported accurately. Finally, of those who reported consuming 'more than usual', according to the \pm 2 SD cut-offs, less than $1 \%$ overreported (i.e. actually did consume more than usual) and 95\% reported accurately (i.e. actually consumed a usual amount), whereas according to the \pm 1 SD cut-offs only $4 \%$ overreported and $53 \%$ reported accurately (i.e. actually consumed a usual amount). The lack of agreement between individuals' self-assessment of usual intake and objective measurements of usual intake further suggests that individuals' self-assessments of usual intake cannot be used to identify and screen out inaccurate reports of dietary intake.

\section{Conclusion}

In conclusion, underreporting of dietary energy intake is widespread and the extent of underreporting can be quite high. Obese subjects and those who practise dietary restraint are two groups that are likely to underreport. Although overreporting is not as prevalent as underreporting, both types of inaccurate reporting should be taken into account when identifying inaccurate energy intake reports. While use of the Goldberg cut-off remains valid, some of its associated limitations may be overcome by the alternative and simpler approach of using pTEE. Group mean values for dietary composition remain fairly similar when either the \pm 1 SD or the \pm 2 SD cut-offs based on the comparison of $\mathrm{rEI}$ with pTEE are selected. In contrast, when examining relationships between diet and health, and in particular dietary composition and energy regulation parameters, use of \pm 1 SD cut-offs may be preferable to the \pm 2 SD cut-offs for excluding inaccurate dietary reports. Finally, individuals' self-assessment of 'usual' intake cannot be used to determine accurate energy intake reports, which are defined as the energy intake required to maintain current body weight according to energy balance principles.

\section{Acknowledgements}

We thank Dr Jerry Dallal for statistical advice and Li Yanping for technical assistance. This research was supported in part by the US Department of Agriculture, Agricultural Research Service, under co-operative agreement \#58-1950-9-001, and by NIH grant \# 5 F32 DK0974703.

\section{References}

1 Schoeller DA, Bandini LG, Dietz WH. Inaccuracies in selfreported intake identified by comparison with the doubly labelled water method. Can. J. Physiol. Pharmacol. 1990; 68: 941-9.

2 Schoeller DA. Limitations in the assessment of dietary energy intake by self-report. Metabolism 1995; 44: 18-22.

3 Bingham SA, Cassidy A, Cole TJ, Welch A, Runswick SA, Black AE, et al. Validation of weighed records and other methods of dietary assessment using the $24 \mathrm{~h}$ urine nitrogen technique and other biological markers. Br. J. Nutr. 1995; 73: 531-50.

4 Briefel RR, Sempos CT, McDowell MA, Chien SC-Y, Alaimo K. Dietary methods research in the third National Health and Nutrition Examination Survey: underreporting of energy intake. Am. J. Clin. Nutr. 1997; 65(Suppl.): 1203S-9S. 
5 Lafay L, Basdevant A, Charles MA, Vray M, Balkau B, Borys $\mathrm{JM}$, et al. Determinants and nature of dietary underreporting in a free-living population: the Fleurbaix Laventie Ville Sante (FLVS) study. Int. J. Obes. 1997; 21: 567-73.

6 Adams SJ. The dietary intake of people with non-insulindependent diabetes (NIDDM): how valid is self-reported intake? J. Hum. Nutr. Diet. 1998; 11: 295-306.

7 Voss S, Kroke A, Klipstein-Grobusch K, Boeing H. Is macronutrient composition of dietary intake data affected by underreporting? Result from the EPIC-Potsdam Study. European Prospective Investigation into Cancer and Nutrition. Eur. J. Clin. Nutr. 1998; 52: 119-26.

8 Pomerleau J, Ostbye T, Bright-See E. Potential underreporting of energy intake in the Ontario Health Survey and its relationship with nutrient and food intakes. Eur. J. Clin. Nutr. 1999; 15: 553-7.

9 Samaras K, Kelly PJ, Campbell LV. Dietary underreporting is prevalent in middle-aged British women and is not related to adiposity (percentage body fat). Int. J. Obes. 1999; 23: $881-8$.

10 Goris AHC, Westerterp-Plantenga MS, Westerterp KR. Undereating and underrecording of habitual food intake in obese men: selective underreporting of fat intake. $\mathrm{Am}$. J. Clin. Nutr. 2000; 71: 130-4.

11 Saltzman E, Roberts SB. The role of energy expenditure in energy regulation: findings from a decade of research. Nutr. Rev. 1995; 53: 209-20.

12 Bray G, Popkin BM. Dietary fat intake does affect obesity! Am.J. Clin. Nutr. 1998; 68: 1157-73.

13 Roberts SB, Pi-Sunyer FX, Dreher M, Hahn R, Hill JO, Kleinman RE, et al. Physiology of fat replacement and fat reduction: effects of dietary fat and fat substitutes on energy regulation. Nutr. Rev. 1998; 56: S29-49.

14 Willett WC. Dietary fat and obesity: an unconvincing relation. Am. J. Clin. Nutr. 1998; 68: 1149-50.

15 Prentice AM, Cole TJ. The Doubly-Labelled Water Method for Measuring Energy Expenditure. Technical Recommendations for Use in Humans: A Consensus Report. Vienna: Section of Nutritional and Health-Related Environmental Studies, International Atomic Energy Agency, International Dietary Energy Consultancy Group, 1990.

16 Speakman JR. Doubly Labelled Water: Theory and Practice, 1st ed. London: Chapman \& Hall, 1997.

17 Black AE. The sensitivity and specificity of the Goldberg cutoff for EI:BMR for identifying diet reports of poor validity. Eur. J. Clin. Nutr. 2000; 54: 395-404.

18 US Department of Agriculture, Agricultural Research Service. CSFII/DHKS 1994-96 Data Set and Documentation: The 1994-96 Continuing Survey of Food Intakes by Individuals and the 1994-96 Diet and Health Knowledge Survey. Springfield, VA: National Technical Information Service, 1998; data tables.

19 Beaton GH, Milner J, Corey P, McGuire V, Cousins M, Stewart $\mathrm{E}$, et al. Sources of variance in 24-hour dietary recall data: implications for nutrition study design and interpretation. Am.J. Clin. Nutr. 1979; 32: 2546-9.

20 Black AE. Critical evaluation of energy intake using the Goldberg cut-off for energy intake:basal metabolic rate. A practical guide to its calculation, use and limitations. Int. J. Obes. 2000; 24: 1119-30.

21 Marr JW, Heady JA. Within- and between-person variation in dietary surveys: number of days needed to classify individuals. Hum. Nutr. Appl. Nutr. 1986; 40A: 347-64.

22 Nelson M, Black AE, Morris JA, Cole TJ. Between- and within-subject variation in nutrient intake from infancy to old age: estimating the number of days required to rank dietary intakes with desired precision. Am. J. Clin. Nutr. 1989; 50: 155-67.

23 Prentice RL. Measurement error and results from analytic epidemiology: dietary fat and breast cancer. J. Natl. Cancer Inst. 1996; 88: 1738-47.

24 Paeratakul S, Popkin BM, Kohlmeier L, Hertz-Picciotto I, Guo X, Edwards LJ. Measurement error in dietary data: implications for the epidemiologic study of the diet-disease relationship. Eur. J. Clin. Nutr. 1998; 52: 722-7.

25 Prentice AM, Black AE, Coward WA, Davies HL, Goldberg GR, Murgatroyd PR, et al. High levels of energy expenditure in obese women. Br. Med. J. 1986; 292: 983-7.

26 Bandini LG, Schoeller DA, Cyr HN, Dietz WH. Validity of reported energy intake in obese and nonobese adolescents. Am.J. Clin. Nutr. 1990; 52: 421-5.

27 Lichtman SW, Pisarska K, Berman ER, Pestone M, Dowling $\mathrm{H}$, Offenbacher E, et al. Discrepancy between self-reported and actual caloric intake and exercise in obese subjects. N. Engl. J. Med. 1992; 327: 1893-8.

28 Bathalon GP, Tucker KL, Hays NP, Vinken AG, Greenberg AS, McCrory MA, et al. Psychological measures of eating behavior and the accuracy of 3 common dietary assessment methods in healthy postmenopausal women. Am. J. Clin. Nutr. 2000; 71: 739-45.

29 Goris AHC, Westerterp KR. Underreporting of habitual food intake is explained by undereating in highly motivated lean women. J. Nutr. 1999; 129: 878-82.

30 Stunkard AJ, Messick S. The three-factor eating questionnaire to measure dietary restraint, disinhibition and hunger. J. Psychosom. Res. 1985; 29: 71-83.

31 Pryer JA, Vrijheid M, Nichols R, Kiggins M, Elliott P. Who are the 'low energy reporters' in the Dietary and Nutritional Survey of British Adults? Int. J. Epidemiol. 1997; 26: $146-54$.

32 Johnson RK, Soultanakis RP, Matthews DE. Literacy and body fatness are associated with underreporting of energy intake in US low-income women using the multiple-pass 24-hour recall: a doubly labeled water study. J. Am. Diet. Assoc. 1998; 98: 1136-40.

33 Braam LA, Ocké MC, Bueno-de-Mesquita HB, Seidell JC. Determinants of obesity-related underreporting of energy intake. Am. J. Epidemiol. 1998; 147: 1081-6.

34 Kretsch MJ, Fong AKH, Green MW. Behavioral and body size correlates of energy intake underreporting by obese and normal-weight women. J. Am. Diet. Assoc. 1999; 99: $300-6$.

35 Tomoyasu NJ, Toth MJ, Poehlman ET. Misreporting of total energy intake in older men and women. J. Am. Geriatr. Soc. 1999; 47: 710-5.

36 Mennen LI, Jackson M, Cade J, Mbanya JC, Lafay L, Sharma S, et al. Underreporting of energy intake in four populations of African origin. Int. J. Obes. 2000; 24: 882-7.

37 Macdiarmid J, Blundell J. Assessing dietary intake: who, what and why of under-reporting. Nutr. Res. Rev. 1998; 11: 231-53.

38 Goldberg GR, Black AE, Jebb SA, Cole TJ, Murgatroyd PR, Coward WA, et al. Critical evaluation of energy intake data using fundamental principles of energy physiology: 1 . Derivation of cut-off limits to identify under-recording. Eur. J. Clin. Nutr. 1991; 45: 569-81.

39 Black AE, Coward WA, Cole TJ, Prentice AM. Human energy expenditure in affluent societies: an analysis of 574 doublylabelled water measurements. Eur. J. Clin. Nutr. 1996; 50: 72-92.

40 Black AE, Goldberg GR, Jebb SA, Livingstone MBE, Cole TJ, Prentice AM. Critical evaluation of energy intake data using fundamental principles of energy physiology: 2. Evaluating the results of published surveys. Eur. J. Clin. Nutr. 1991; 45: 583-99.

41 Warwick PM, Baines J. Energy expenditure in free-living smokers and nonsmokers: comparison between factorial, intake-balance, and doubly labeled water measures. Am. J. Clin. Nutr. 1996; 63: 15-21. 
42 FAO/WHO/UNU. Energy and Protein Requirements: Report of a Joint FAO/WHO/UNU Expert Consultation. Geneva: World Health Organization, 1985.

43 Vinken AG, Bathalon GP, Sawaya AL, Dallal GE, Tucker KL, Roberts SB. Equations for predicting the energy requirements of healthy adults aged 18-81y. Am. J. Clin. Nutr. 1999; 69: 920-6.

44 Shetty PS, Henry CJK, Black AE, Prentice AM. Energy requirements of adults: an update on basal metabolic rates (BMRs) and physical activity levels (PALs). Eur.J. Clin. Nutr. 1996; 50(Suppl. 1): S11-23.

45 Roberts SB, Heyman MB, Evans WJ, Fuss P, Tsay R, Young VR. Dietary energy requirements of young adult men, determined by using the doubly labeled water method. $\mathrm{Am}$. J. Clin. Nutr. 1991; 54: 499-505.

46 Roberts SB, Young VR, Fuss P, Heyman MB, Fiatarone M,
Dallal GE, et al. What are the dietary energy needs of elderly adults? Int. J. Obes. 1992; 16: 969-76.

47 Prentice AM, Black AE, Coward WA, Cole TJ. Energy expenditure in overweight and obese adults in affluent societies: an analysis of 319 doubly-labelled water measurements. Eur. J. Clin. Nutr. 1996; 50: 93-7.

48 Goldberg GR. From individual variation in energy intakes ...to variations in energy requirements and adaptations to them. Br. J. Nutr. 1997; 78(Suppl. 2): S81-94.

49 Macdiarmid JI, Vail A, Cade JE, Blundell JE. The sugar-fat relationship revisited: differences in consumption between men and women of varying BMI. Int. J. Obes. 1998; 22: 1053-61.

50 Stallone DD, Brunner EJ, Bingham SA, Marmot MG. Dietary assessment in Whitehall II: the influence of reporting bias on apparent socioeconomic variation in nutrient intakes. Eur. J. Clin. Nutr. 1997; 51: 815-25. 